\title{
BIODIVERSITY OF WEED COMMUNITIES IN ORGANIC AND CONVENTIONAL ORCHARDS
}

\author{
Jerzy LISEK*, Lidia SAS-PASZT \\ Research Institute of Horticulture \\ Konstytucji 3 Maja 1/3, 96-100 Skierniewice, Poland \\ Received: February 2015; Accepted: May 2015 \\ Edited by B. Matysiak
}

\begin{abstract}
The aim of the study was to analyze the occurrence of segetal and ruderal weeds in young apple and cherry organic orchards, where weeds under tree canopies were controlled mechanically with a rototiller, and in conventional orchards, where post-emergence herbicides were used. The research material consisted of phytosociological relevés recorded according to the Braun-Blanquet method, in three organic and three conventional orchards, situated near Skierniewice (central Poland), in 2010-2013. On the basis of the collected source material, species composition for the synanthropic flora, as well as phytosociological stability, the cover factor, and weed infestation rate for the analysed species were determined. The total number of the recorded weeds and the average number of weed species in a single relevé were for each of the three organic orchards greater than for the conventional orchards. The organic orchard situated in the same location as the conventional orchard was characterized by a greater biodiversity of flora. Systematic shallow cultivation of the soil, without herbicide treatments, resulted in the proliferation of perennial weeds.
\end{abstract}

Key words: synanthropic flora, farming systems, apple and sour cherry trees, weed species

\section{INTRODUCTION}

The factors that modify the biodiversity of synanthropic flora in agricultural and horticultural crops are the environmental conditions and soil maintenance method. Research conducted in cereals and vegetables plots has shown that the number of weeds and their relative proportions vary depending on the forecrop, the species being cultivated, crop rotation, the time and number of cultivation treatments, and the type of tools, herbicides and mulches used (Derksen et al. 1993; Błażewicz-Woźniak 2004; Anyszka \& Kohut 2011). In winter wheat, the cause of a reduction in biodiversity is systematic, simplified cultivation of the soil (Sekutowski \& Domaradzki 2009) as well as monoculture and the use of herbicides (Jędruszczak \& Antoszek 2004). Papers dealing with the differences in weed biodiversity between organic and conventional crops concern, for the majority of arable fields, mostly cereals and root crops (Moreby et al. 1994; Albrecht \& Mattheis 1998; Hald 1999; Elsen 2000; Rydberg \& Milberg 2000; Hyvönen et al. 2003; Hole et al. 2005). The authors of most of them state that weed communities in organic crops are floristically more varied than those in conventional crops. No significant differences have been found in the total number of weed species between organic and conventional cultivation of root crops (Kuźniewski \& JezierskaDomaradzka 2007) and beans (Dobrzański \& Pałczyński 2007). The main method of maintaining the soil under the crowns of trees in commercial orchards is still the use of herbicides, which determine the specific species composition of weed infestation (Wróbel 1999; Lipecki 2004; Lisek 2012), often of a cosmopolitan character (Mashaly \& Awad 2003; Ustuner \& Ustuner 2011). The literature on the species composition of weeds in organic orchards is scarce. According to Tasseva (2005) the diversity of weeds in organic orchards is greater than in conven- 
tional orchards and the species composition of their agrophytocoenoses can be different (Mika 2004). The authors of papers on the maintenance of soil in organic orchards focus primarily on the growth and yielding of trees, fruit quality, and the physicochemical properties of the soil, remarking only difficulties in controlling certain weed species, for example, Agropyron repens (Stefanelli et al. 2009; Rabcewicz \& Białkowski 2011; Granatstein et al. 2014). Rabcewicz \& Białkowski (2011), while assessing the effectiveness of controlling orchard weeds with a rototiller, reported that annual weeds were controlled more effectively than the perennial ones, but did not provide species composition of the infestation. The diversity of weeds affects the biodiversity of microorganisms, invertebrates, and birds (Marshall et al. 2003).

The aim of the study was to evaluate and compare the communities of weeds in organic and conventional apple and sour cherry orchards, and to identify weed species that can be a problem in the increasing number of new organic orchards.

\section{MATERIALS AND METHODS}

\section{Orchards and environmental conditions}

Phytosociological studies were conducted in three organic orchards in the localities of Dąbrowice, Mokra Lewa, and Maków, and in three conventional orchards in Dąbrowice, Julków and Ostrowiec, situated within a $20 \mathrm{~km}$ radius from Skierniewice (central Poland) and established in 2008. In the experimental orchard in Dąbrowice, owned by the Research Institute of Horticulture, the organic and conventional orchards were adjacent to each other. The organic orchards were comprised of apple trees ('Topaz'/M 26, 'Ariwa'/M 26) and sour cherry trees ('Sabina' and 'Debreceni Botermo', both on Prunus mahaleb seedlings), growing at a spacing of $4 \times 2.5 \mathrm{~m}$. The conventional orchards were comprised of apple trees ('Jonagold'/M26) and sour cherry trees ('Schattenmorelle' on Prunus mahaleb seedlings), planted at the same or similar $(4 \times 2 \mathrm{~m})$ spacing. The orchards were situated on flatland (only the organic orchards in Mokra Lewa and Maków were situated on a slight slope, with a 1$2 \%$ gradient), on a pseudopodsolic soil of quality class IV or V, slightly acidic (pH 5.2-6.9). The soil had the following mechanical composition - sand: $63-84 \%$; silt: $12-35 \%$; clay: $1-8 \%$, and the organic matter content was $1.1-1.9 \%$. The orchards in Dąbrowice, Julków and Ostrowiec were established on a land occupied by intensive fruit crops cultivated for at least 30 years, with a 2-3 year break for forecrops (cereals, mustard). The organic orchards in Mokra Lewa and Maków were established after short-lived crops (cereals, mustard, fruit nurseries in rotation).

\section{Weed management}

From the time of establishing the organic orchards, the soil under tree canopies, in $1.5 \mathrm{~m}$ wide strips, had been cultivated using an automatic retracting tree rotary weeder, 3-4 treatments per year, the first one in the second half of May, and the last one in August/September. The soil was tilled to a depth of $5-8 \mathrm{~cm}$. In the conventional orchards, during the growing season, 3-4 treatments were performed with foliar herbicides: at least 2 treatments with glyphosate, one treatment with 2-methyl-4chlorophenoxyacetic acid (MCPA), and one treatment with glufosinate-ammonium in some orchards and seasons. The inter-rows of the conventional orchards and the organic orchard in Dąbrowice were grassed over, whereas in the organic orchards in Mokra Lewa and Maków were cultivated with a rototiller.

\section{Phytosociological relevés}

In 2010-2013, 240 phytosociological relevés (60 per year) were recorded from a surface area of 0.8 ha in each of the orchards ( 0.4 ha apple and 0.4 ha sour cherry trees), prior to weed control treatments, according to the Braun-Blanquet method (1964). Half of the relevés, recorded in the second half of May, documented primary weed infestation. The other half of the relevés were recorded in the second half of August and documented secondary weed infestation. The relevés covered an area of $30 \mathrm{~m}^{2}$ $(20 \times 1.5 \mathrm{~m})$ of the soil under the tree crowns. Phytosociological stability and the cover factor were calculated for each weed species, and on the basis of these data weed infestation rate was determined.

Phytosociological stability (S) was expressed on a 5-point scale with an additional subclass. The stability classes according to the percentage of relevés in which a given species was found were as 
follows: $5-81-100 \%, 4-61-80 \%, 3-41-60 \%$, $2-21-40 \%, 1-2-20 \%, \pm<1 \%$ for all the relevés.

The cover factor (CF) represents the sum of the average percentages of soil coverage by a given species in all the relevés in which that species occurs/the total number of relevés $\times 100$. By adding the cover factors for the various short-lived species and the perennials, the percentage share of these two groups in covering the soil with weeds was determined.

Weed infestation rates were as follow:

I - very high: $S=5$ or $4, C F>1000$;

II - high: $S=5$ or $4, C F=501-1000$ or $S=3, C F>750$;

III - moderate: $\mathrm{S}=5$ or $4, \mathrm{CF}=251-500$ or $\mathrm{S}=3$, $\mathrm{CF}=501-750$;

IV - low: $\mathrm{S}=5$ or $4, \mathrm{CF}=51-250$ or $\mathrm{S}=3, \mathrm{CF}=251-500$; $\mathrm{V}$ - sporadic: other lower stability classes and cover factors.

The data obtained from the relevés were analysed separately for each of the orchards under assessment.

Biodiversity of weed communities was compared for the organic orchard and the conventional orchard with apple trees in Dąbrowice with the following indices:

Shannon-Wiener's biodiversity index - H' (Shannon 1948)

$$
\mathrm{H}^{\prime}=-\sum_{\mathrm{i}=1}^{s} p_{i} \ln p_{i}
$$

Simpson's dominance index - D (Simpson 1949)

$$
\mathrm{D}=\sum_{i=1}^{s} p_{i}{ }^{2}
$$

where:

$p_{i}=n_{i} / N, n_{i}-$ number of individuals of the $i$-th species, $\mathrm{N}$ - total number of individuals; $\mathrm{S}$ - number of species.

The Shannon-Wiener biodiversity index (H') increases with the number of weed species in the community and the degree of equalization in their numbers. The Simpson dominance index (D) takes on values in the range from 0 to 1 , and when the value reaches 1 , it means there is no diversity (a single-species community). The bigger value of Simpson dominance index (D) means the lower biodiversity. The data on the number of each species, necessary for the calculation of the indices, were collected in midMay, in randomly selected but evenly distributed locations in the apple orchard, from 12 squares with an area of $0.25 \mathrm{~m}^{2}(0.5 \times 0.5 \mathrm{~m})$ each, and converted to a surface area of $1 \mathrm{~m}^{2}$. The indices are presented as an average for four years.

\section{RESULTS}

The species composition of weed infestation in apple and sour cherry orchards did not differ, therefore data are presented together. The most important short-lived weed species occurring at rate I or II in all three organic orchards were Echinochloa crusgalli and Chenopodium album (Tables 1, 2, 3). Other annual species occurred at a very high or high rate in one of the organic orchards - Amaranthus retroflexus, Stellaria media, Geranium pusillum in Dąbrowice, and Polygonum persicaria and Setaria pumila in Mokra Lewa. Among the perennial weeds, Agropyron repens occurred at rate I or II in two organic orchards (Mokra Lewa and Maków), and Equisetum arvense in one of the orchards (Mokra Lewa).

The short-lived species: Stellaria media, Poa annua and Capsella bursa-pastoris occurred at rate I or II in three conventional orchards, Chenopodium album and Echinochloa crus-galli in two (Ostrowiec and Julków), and Geranium pusillum in one (Julków) (Tables 4, 5, 6). The perennial species Equisetum arvense and Taraxacum officinale (Ostrowiec), and Epilobium adenocaulon (Julków) heavily infested two conventional orchards.

The total number of the recorded weeds and the average number of weed species in a single relevé were for each of the three organic orchards greater than for the conventional orchards (Table 7). The share of short-lived weeds in covering the soil was much higher than the share of perennial weeds in the three conventional orchards and in one organic orchard - in Dąbrowice.

The value of the Shannon-Wiener index was higher, and the value of the Simpson index was lower for the organic orchard compared with the conventional orchard, which means that the weed community in the organically grown part of the orchard in Dąbrowice was characterized by a greater species diversity (biodiversity) than in the conventionally grown part of that orchard (Table 7). 
Table 1. Biodiversity of weed communities in the organic orchard Dąbrowice, as an average for four years (2010-2013)

\begin{tabular}{|c|c|c|c|}
\hline Species & $\begin{array}{c}\text { Phytosociological } \\
\text { stability }(\mathrm{S})\end{array}$ & Cover factor $(\mathrm{CF})$ & $\begin{array}{c}\text { Weed infestation } \\
\text { rate }\end{array}$ \\
\hline \multicolumn{4}{|l|}{ Short-lived } \\
\hline Echinochloa crus-galli (L.) P. Beauv. & 5 & 1582 & $\mathrm{I}$ \\
\hline Chenopodium album $\mathrm{L}$. & 5 & 1509 & $\mathrm{I}$ \\
\hline Amaranthus retroflexus $\mathrm{L}$. & 4 & 511 & II \\
\hline Stellaria media (L.) Vill. & 4 & 505 & II \\
\hline Geranium pusillum Burm. F. ex L. & 4 & 503 & II \\
\hline Capsella bursa-pastoris (L.) Med. & 4 & 392 & III \\
\hline Bromus hordeaceus L. & 4 & 386 & III \\
\hline Bromus tectorum $\mathrm{L}$. & 4 & 295 & III \\
\hline Erodium cicutarium (L.) L’Hér. & 3 & 256 & IV \\
\hline Vicia villosa Roth. & 2 & 165 & $\mathrm{~V}$ \\
\hline Setaria viridis (L.) P. Beauv. & 3 & 132 & $\mathrm{~V}$ \\
\hline Digitaria sanguinalis (L.) Scop. & 2 & 119 & $\mathrm{~V}$ \\
\hline Poa anпua $\mathrm{L}$. & 3 & 105 & $\mathrm{~V}$ \\
\hline Conyza canadensis (L.) Cronq. & 3 & 104 & $\mathrm{~V}$ \\
\hline Galinsoga parviflora Cav. & 3 & 98 & $\mathrm{~V}$ \\
\hline Polygonum aviculare L. & 4 & 95 & $\mathrm{~V}$ \\
\hline Atriplex patulum L. & 2 & 89 & $\mathrm{~V}$ \\
\hline Polygonum persicaria $\mathrm{L}$. & 2 & 82 & $\mathrm{~V}$ \\
\hline Senecio vulgaris L. & 3 & 76 & $\mathrm{~V}$ \\
\hline Viola arvensis Murr. & 3 & 72 & $\mathrm{~V}$ \\
\hline Arenaria serpyllifolia $\mathrm{L}$. & 2 & 58 & $\mathrm{~V}$ \\
\hline Crepis capillaris (L.) Wallr. & 1 & 56 & $\mathrm{~V}$ \\
\hline Crepis biennis $\mathrm{L}$. & 1 & 52 & $\mathrm{~V}$ \\
\hline Hypochoeris glabra L. & 1 & 48 & $\mathrm{~V}$ \\
\hline Veronica arvensis $\mathrm{L}$. & 2 & 42 & $\mathrm{~V}$ \\
\hline Matricaria maritima L. ssp. inodora & 2 & 39 & $\mathrm{~V}$ \\
\hline Chamomilla suaveolens (Pursh) Rydb. & 1 & 36 & $\mathrm{~V}$ \\
\hline Myosotis stricta Lk. & 1 & 26 & $\mathrm{~V}$ \\
\hline Spergularia rubra (L.) Presl. & 1 & 24 & $\mathrm{~V}$ \\
\hline Cerastium holosteoides Fr. em. Hyl. & 1 & 19 & $\mathrm{~V}$ \\
\hline \multicolumn{4}{|l|}{ Perennial } \\
\hline Convolvulus arvensis $\mathrm{L}$. & 3 & 459 & IV \\
\hline Agropyron repens L. & 3 & 346 & IV \\
\hline Taraxacum officinale F.H. Wigg. & 4 & 329 & III \\
\hline Equisetum arvense L. & 2 & 298 & IV \\
\hline Rumex crispus L. & 2 & 118 & $\mathrm{~V}$ \\
\hline Epilobium adenocaulon Hausskn. & 2 & 108 & $\mathrm{~V}$ \\
\hline Rorippa sylvestris (L.) Besser & 2 & 106 & $\mathrm{~V}$ \\
\hline Cirsium arvense (L.) Scop. & 1 & 38 & $\mathrm{~V}$ \\
\hline
\end{tabular}


Table 2. Biodiversity of weed communities in organic orchard Maków, as an average for four years (2010-2013)

\begin{tabular}{|c|c|c|c|}
\hline Species & $\begin{array}{l}\text { Phytoso- } \\
\text { ciological } \\
\text { stability } \\
\text { (S) }\end{array}$ & $\begin{array}{l}\text { Cover } \\
\text { factor } \\
(\mathrm{CF})\end{array}$ & $\begin{array}{c}\text { Weed } \\
\text { infesta- } \\
\text { tion rate }\end{array}$ \\
\hline \multicolumn{4}{|l|}{ Short-lived } \\
\hline Chenopodium album $\mathrm{L}$. & 5 & 1068 & I \\
\hline Echinochloa crus-galli (L.) P. Beauv. & 4 & 793 & II \\
\hline Raphanus raphanistrum $\mathrm{L}$. & 3 & 620 & III \\
\hline Matricaria maritima L. ssp. inodora (L.) & 3 & 367 & IV \\
\hline Polygonum lapathifolium L. & 3 & 261 & IV \\
\hline Polygonum persicaria $\mathrm{L}$. & 3 & 254 & IV \\
\hline Amaranthus retroflexus $\mathrm{L}$. & 2 & 126 & $\mathrm{~V}$ \\
\hline Роа аппиа $\mathrm{L}$. & 2 & 115 & $\mathrm{~V}$ \\
\hline Fallopia convolvulus (L.) Á. Löve & 2 & 111 & $\mathrm{~V}$ \\
\hline Digitaria sanguinalis (L.) Scop. & 2 & 106 & $\mathrm{~V}$ \\
\hline Conyza canadensis (L.) Cronq. & 2 & 106 & $\mathrm{~V}$ \\
\hline Erodium cicutarium (L.) L’Hér. & 2 & 97 & $\mathrm{~V}$ \\
\hline Spergula arvensis L. & 2 & 93 & $\mathrm{~V}$ \\
\hline Stellaria media (L.) Vill. & 2 & 91 & $\mathrm{~V}$ \\
\hline Scleranthus annuus L. & 1 & 88 & $\mathrm{~V}$ \\
\hline Capsella bursa-pastoris (L.) Med. & 2 & 63 & $\mathrm{~V}$ \\
\hline Vicia villosa Roth. & 2 & 58 & $\mathrm{~V}$ \\
\hline Euphorbia helioscopia L. & 2 & 51 & $\mathrm{~V}$ \\
\hline Galinsoga parviflora Cav. & 2 & 48 & $\mathrm{~V}$ \\
\hline Senecio vulgaris $\mathrm{L}$. & 3 & 45 & $\mathrm{~V}$ \\
\hline Sonchus asper (L.) Hill & 3 & 32 & $\mathrm{~V}$ \\
\hline Centaurea cyanus $\mathrm{L}$. & 2 & 31 & $\mathrm{~V}$ \\
\hline Geranium pusillum Burm. F. ex L. & 1 & 26 & $\mathrm{~V}$ \\
\hline Papaver rhoeas $\mathrm{L}$. & 1 & 22 & $\mathrm{~V}$ \\
\hline Bromus hordeaceus L. & 2 & 19 & $\mathrm{~V}$ \\
\hline Thlaspi arvense L. & 1 & 16 & $\mathrm{~V}$ \\
\hline Spergularia rubra (L.) Presl. & 1 & 14 & $\mathrm{~V}$ \\
\hline Lactuca serriola $\mathrm{L}$. & + & 8 & $\mathrm{~V}$ \\
\hline Gypsophila muralis L. & + & 6 & $\mathrm{~V}$ \\
\hline Consolida regalis S.F. Gray & + & 5 & $\mathrm{~V}$ \\
\hline
\end{tabular}

\begin{tabular}{|c|c|c|c|}
\hline Perennial & & & \\
\hline Agropyron repens $\mathrm{L}$. & 5 & 1984 & I \\
\hline Cirsium arvense (L.) Scop. & 3 & 673 & III \\
\hline Rumex acetosella L. & 3 & 529 & III \\
\hline Equisetum arvense $\mathrm{L}$. & 3 & 358 & IV \\
\hline Artemisia vulgaris $\mathrm{L}$. & 3 & 314 & IV \\
\hline Sonchus arvensis $\mathrm{L}$. & 2 & 306 & $\mathrm{~V}$ \\
\hline Rumex crispus L. & 1 & 86 & $\mathrm{~V}$ \\
\hline Trifolium repens $\mathrm{L}$. & 2 & 82 & V \\
\hline Tussilago farfara $\mathrm{L}$. & 1 & 78 & $\mathrm{~V}$ \\
\hline Trifolium arvense $\mathrm{L}$. & 1 & 76 & $\mathrm{~V}$ \\
\hline Taraxacum officinale $\mathrm{F}$. H. Wigg. & 2 & 69 & $\mathrm{~V}$ \\
\hline Convolvulus arvensis $\mathrm{L}$. & 1 & 63 & $\mathrm{~V}$ \\
\hline Hypochoeris radicata $\mathrm{L}$. & 1 & 51 & $\mathrm{~V}$ \\
\hline Centaurea jacea $\mathrm{L}$. & + & 9 & $\mathrm{~V}$ \\
\hline
\end{tabular}

Table 3. Biodiversity of weed communities in organic orchard Mokra Lewa, as an average for four years (2010-2013)

\begin{tabular}{|c|c|c|c|}
\hline Species & $\begin{array}{l}\text { Phytoso- } \\
\text { ciological } \\
\text { stability } \\
\text { (S) }\end{array}$ & $\begin{array}{c}\text { Cover } \\
\text { factor } \\
(\mathrm{CF})\end{array}$ & $\begin{array}{l}\text { Weed } \\
\text { infesta- } \\
\text { tion rate }\end{array}$ \\
\hline \multicolumn{4}{|l|}{ Short-lived } \\
\hline Echinochloa crus-galli (L.) P. Beauv. & 5 & 1006 & $\mathrm{I}$ \\
\hline Polygonum persicaria $\mathrm{L}$. & 3 & 756 & II \\
\hline Chenopodium album $\mathrm{L}$. & 5 & 702 & II \\
\hline Setaria pumila (Poir.) Roem. et Schult. & 4 & 559 & II \\
\hline Matricaria maritima L. ssp. inodora (L.) & 3 & 187 & $\mathrm{~V}$ \\
\hline Viola arvensis Murr. & 2 & 101 & $\mathrm{~V}$ \\
\hline Raphanus raphanistrum $\mathrm{L}$. & 2 & 99 & $\mathrm{~V}$ \\
\hline Conyza canadensis (L.) Cronq. & 2 & 97 & $\mathrm{~V}$ \\
\hline Polygonum aviculare $\mathrm{L}$. & 2 & 96 & $\mathrm{~V}$ \\
\hline Fallopia convolvulus (L.) A. Löve & 2 & 72 & $\mathrm{~V}$ \\
\hline Sinapis arvensis $\mathrm{L}$. & 2 & 68 & $\mathrm{~V}$ \\
\hline Anthemis arvensis $\mathrm{L}$. & 2 & 66 & $\mathrm{~V}$ \\
\hline Spergula arvensis $\mathrm{L}$. & 2 & 58 & $\mathrm{~V}$ \\
\hline Senecio vulgaris $\mathrm{L}$ & 2 & 56 & V \\
\hline Capsella bursa-pastoris (L.) Med. & 2 & 52 & $\mathrm{~V}$ \\
\hline Pоа аппиа $\mathrm{L}$. & 2 & 48 & $\mathrm{~V}$ \\
\hline Polygonum lapathifolium L. & 2 & 46 & $\mathrm{~V}$ \\
\hline Vicia villosa Roth. & 2 & 45 & $\mathrm{~V}$ \\
\hline Gnaphalium uliginosum L. & 1 & 42 & $\mathrm{~V}$ \\
\hline Stellaria media (L.) Vill. & 2 & 41 & $\mathrm{~V}$ \\
\hline Vicia hirsuta (L.) S.F. Gray & 1 & 39 & $\mathrm{~V}$ \\
\hline Lamium amplexicaule $\mathrm{L}$. & 1 & 38 & $\mathrm{~V}$ \\
\hline Geranium pusillum Burm. F. ex L. & 1 & 37 & $\mathrm{~V}$ \\
\hline Galinsoga parviflora Cav. & 2 & 32 & $\mathrm{~V}$ \\
\hline Galeopsis tetrahit $\mathrm{L}$. & 1 & 31 & $\mathrm{~V}$ \\
\hline Myosotis stricta $\mathrm{Lk}$. & 2 & 30 & $\mathrm{~V}$ \\
\hline Spergularia rubra (L.) Presl. & 2 & 29 & $\mathrm{~V}$ \\
\hline Erigeron acer $\mathrm{L}$. & 1 & 28 & $\mathrm{~V}$ \\
\hline Erigeron ramosus (Walt.) B.S.P. & 1 & 22 & $\mathrm{~V}$ \\
\hline Erigeron annuus (L.) Pers. & 1 & 21 & $\mathrm{~V}$ \\
\hline Senecio jacobaea L. & 1 & 19 & V \\
\hline Polygonum hydropiper L. & 1 & 17 & $\mathrm{~V}$ \\
\hline Lactuca serriola $\mathrm{L}$. & 1 & 14 & $\mathrm{~V}$ \\
\hline Anagallis arvensis L. & + & 8 & $\mathrm{~V}$ \\
\hline \multicolumn{4}{|l|}{ Perennial } \\
\hline Equisetum arvense $\mathrm{L}$. & 5 & 1368 & $\mathrm{I}$ \\
\hline Agropyron repens $\mathrm{L}$. & 4 & 897 & II \\
\hline Rumex acetosella $\mathrm{L}$. & 3 & 680 & III \\
\hline Artemisia vulgaris $\mathrm{L}$. & 3 & 510 & III \\
\hline Cirsium arvense (L.) Scop. & 3 & 426 & IV \\
\hline Convolvulus arvensis L. & 2 & 394 & V \\
\hline Rorippa sylvestris (L.) Besser & 2 & 282 & $\mathrm{~V}$ \\
\hline Rumex crispus $\mathrm{L}$. & 2 & 156 & $\mathrm{~V}$ \\
\hline Stachys palustris L. & 1 & 59 & $\mathrm{~V}$ \\
\hline Potentilla anserina $\mathrm{L}$. & 1 & 52 & $\mathrm{~V}$ \\
\hline Potentilla reptans $\mathrm{L}$. & 1 & 49 & $\mathrm{~V}$ \\
\hline Epilobium adenocaulon Hausskn. & 2 & 48 & V \\
\hline Hieracium pilosella $\mathrm{L}$ & 1 & 45 & $\mathrm{~V}$ \\
\hline Plantago major L. & 2 & 41 & $\mathrm{~V}$ \\
\hline Ranunculus repens $\mathrm{L}$. & 1 & 39 & $\mathrm{~V}$ \\
\hline Tanacetum vulgare $\mathrm{L}$. & 1 & 31 & $\mathrm{~V}$ \\
\hline Mentha arvensis L. & 1 & 30 & $\mathrm{~V}$ \\
\hline Heracleum sphondylium $\mathrm{L}$. & 1 & 28 & $\mathrm{~V}$ \\
\hline Tussilago farfara $\mathrm{L}$. & 1 & 27 & $\mathrm{~V}$ \\
\hline Taraxacum officinale F.H. Wigg. & 1 & 22 & $\mathrm{~V}$ \\
\hline Solidago gigantea Aiton & 1 & 19 & V \\
\hline Anthoxanthum odoratum $\mathrm{L}$. & 1 & 18 & $\mathrm{~V}$ \\
\hline Carex hirta $\mathrm{L}$. & 1 & 16 & $\mathrm{~V}$ \\
\hline Hypochoeris radicata $\mathrm{L}$. & 1 & 14 & $\mathrm{~V}$ \\
\hline Leontodon hispidus L. & 1 & 13 & $\mathrm{~V}$ \\
\hline Holcus mollis L. & 1 & 12 & $\mathrm{~V}$ \\
\hline Dactylis glomerata L. & 1 & 11 & V \\
\hline Alopecurus pratensis L. & 1 & 10 & $\mathrm{~V}$ \\
\hline Knautia arvensis (L.) Coult. & + & 8 & $\mathrm{~V}$ \\
\hline
\end{tabular}


Table 4. Biodiversity of weed communities in conventional orchard Dąbrowice, as an average for four years (2010-2013)

\begin{tabular}{|c|c|c|c|}
\hline Species & $\begin{array}{c}\text { Phytosociological } \\
\text { stability }(\mathrm{S})\end{array}$ & Cover factor $(\mathrm{CF})$ & Weed infestation rate \\
\hline \multicolumn{4}{|l|}{ Short-lived } \\
\hline Stellaria media (L.) Vill. & 5 & 2250 & I \\
\hline Capsella bursa-pastoris (L.) Med. & 5 & 1016 & I \\
\hline Роа аппиа $\mathrm{L}$ & 5 & 768 & II \\
\hline Chenopodium album $\mathrm{L}$. & 4 & 701 & III \\
\hline Echinochloa crus-galli (L.) P. Beauv. & 4 & 699 & III \\
\hline Geranium pusillum Burm. F. ex L. & 4 & 642 & III \\
\hline Polygonum aviculare $\mathrm{L}$. & 4 & 423 & III \\
\hline Amaranthus retroflexus $\mathrm{L}$. & 3 & 262 & IV \\
\hline Lamium purpureum $\mathrm{L}$. & 4 & 245 & IV \\
\hline Polygonum persicaria $\mathrm{L}$. & 2 & 167 & $\mathrm{~V}$ \\
\hline Bromus hordeaceus L. & 2 & 153 & $\mathrm{~V}$ \\
\hline Fallopia convolvulus (L.) A. Löve & 2 & 148 & $\mathrm{~V}$ \\
\hline Matricaria maritima L. ssp. inodora & 2 & 121 & $\mathrm{~V}$ \\
\hline Conyza canadensis (L.) Cronq. & 2 & 119 & $\mathrm{~V}$ \\
\hline Viola arvensis Murr. & 2 & 105 & $\mathrm{~V}$ \\
\hline Senecio vulgaris $\mathrm{L}$. & 2 & 95 & $\mathrm{~V}$ \\
\hline Urtica urens $\mathrm{L}$. & 1 & 83 & $\mathrm{~V}$ \\
\hline Lamium roseum $\mathrm{L}$. & 3 & 59 & $\mathrm{~V}$ \\
\hline Myosurus minimus L. & 1 & 45 & $\mathrm{~V}$ \\
\hline \multicolumn{4}{|l|}{ Perennial } \\
\hline Equisetum arvense $\mathrm{L}$. & 5 & 272 & III \\
\hline Taraxacum officinale F. H. Wigg. & 5 & 263 & III \\
\hline Epilobium adenocaulon Hausskn. & 5 & 231 & IV \\
\hline Convolvulus arvensis $\mathrm{L}$. & 2 & 148 & $\mathrm{~V}$ \\
\hline Cirsium arvense (L.) Scop. & 1 & 79 & $\mathrm{~V}$ \\
\hline
\end{tabular}

Table 5. Biodiversity of weed communities in conventional orchard Julków, as an average for four years (2010-2013)

\begin{tabular}{lccc}
\hline \multicolumn{1}{c}{ Species } & $\begin{array}{c}\text { Phytosociological } \\
\text { stability (S) }\end{array}$ & Cover factor (CF) & Weed infestation rate \\
\hline Short-lived & 5 & & I \\
\hline Geranium pusillum Burm. F. ex L. & 4 & 1120 & I \\
Chenopodium album L. & 4 & 1003 & I \\
Echinochloa crus -galli (L.) P. Beauv. & 5 & 891 & II \\
Stellaria media (L.) Vill. & 5 & 754 & II \\
Poa annua L. & 5 & 692 & II \\
Capsella bursa-pastoris (L.) Med. & 3 & 495 & IV \\
Arabidopsis thaliana (L.) Heynh. & 5 & 198 & IV \\
Lamium purpureum L. & 3 & 252 & IV \\
Galinsoga parviflora Cav. & 4 & 147 & IV \\
Bromus hordeaceus L. & 3 & 123 & V \\
Senecio vulgaris L. & 2 & 121 & V \\
Polygonum aviculare L. & 2 & 119 & V \\
Myosurus minimus L. & 2 & 108 & V \\
Erophila verna (L.) Chevall. & 2 & 105 & II \\
Fallopia convolvulus (L.) A. Löve & & & III \\
\hline Perennial & 5 & 596 & V \\
\hline Epilobium adenocaulon Hausskn. & 5 & 398 & 114 \\
Taraxacum officinale F. H. Wigg. & 2 & & \\
Trifolium repens L. & & & \\
\hline
\end{tabular}


Table 6. Biodiversity of weed communities in conventional orchard, Ostrowiec, as an average for four years (2010-2013)

\begin{tabular}{|c|c|c|c|}
\hline Species & $\begin{array}{l}\text { Phytosociological } \\
\text { stability }(\mathrm{S})\end{array}$ & Cover factor $(\mathrm{CF})$ & Weed infestation rate \\
\hline \multicolumn{4}{|l|}{ Short-lived } \\
\hline Echinochloa crus-galli (L.) P. Beauv. & 5 & 1484 & I \\
\hline Stellaria media (L.) Vill. & 5 & 1256 & I \\
\hline Capsella bursa-pastoris (L.) Med. & 5 & 1126 & I \\
\hline Chenopodium album $\mathrm{L}$. & 5 & 782 & II \\
\hline Pоа аппиа $\mathrm{L}$. & 5 & 541 & II \\
\hline Geranium pusillum Burm. F. ex L. & 5 & 476 & III \\
\hline Amaranthus retroflexus L. & 3 & 166 & $\mathrm{~V}$ \\
\hline Lamium purpureum $\mathrm{L}$. & 4 & 160 & IV \\
\hline Galinsoga parviflora Cav. & 3 & 154 & $\mathrm{~V}$ \\
\hline Urtica urens $\mathrm{L}$. & 3 & 152 & $\mathrm{~V}$ \\
\hline Bromus hordeaceus L. & 3 & 147 & $\mathrm{~V}$ \\
\hline Conyza canadensis (L.) Cronq. & 4 & 142 & $\mathrm{~V}$ \\
\hline Viola arvensis Murr. & 4 & 131 & $\mathrm{~V}$ \\
\hline Polygonum aviculare L. & 4 & 128 & $\mathrm{~V}$ \\
\hline Lamium roseum $\mathrm{L}$. & 3 & 124 & $\mathrm{~V}$ \\
\hline Senecio vulgaris $\mathrm{L}$. & 4 & 115 & $\mathrm{~V}$ \\
\hline Fallopia convolvulus (L.) A. Löve & 3 & 106 & $\mathrm{~V}$ \\
\hline Polygonum persicaria $\mathrm{L}$. & 3 & 97 & $\mathrm{~V}$ \\
\hline Veronica persica Poir. & 3 & 92 & $\mathrm{~V}$ \\
\hline Matricaria maritima L. ssp. inodora & 2 & 81 & $\mathrm{~V}$ \\
\hline Sinapis arvensis $\mathrm{L}$. & 2 & 78 & $\mathrm{~V}$ \\
\hline \multicolumn{4}{|l|}{ Perennial } \\
\hline Equisetum arvense $\mathrm{L}$. & 5 & 580 & II \\
\hline Taraxacum officinale F. H. Wigg. & 5 & 532 & II \\
\hline Epilobium adenocaulon Hausskn. & 5 & 302 & III \\
\hline Cirsium arvense (L.) Scop. & 3 & 146 & $\mathrm{~V}$ \\
\hline Convolvulus arvensis $\mathrm{L}$. & 3 & 139 & $\mathrm{~V}$ \\
\hline Trifolium repens $\mathrm{L}$. & 2 & 98 & $\mathrm{~V}$ \\
\hline Rorippa sylvestris (L.) Besser & 2 & 96 & $\mathrm{~V}$ \\
\hline
\end{tabular}

Table 7. Biodiversity of weed communities in organic and conventional orchards as an average for four years (2010-2013)

\begin{tabular}{lrrrrrr}
\hline \multirow{2}{*}{\multicolumn{1}{c}{ Feature }} & \multicolumn{3}{c}{ Organic orchards - locality } & \multicolumn{3}{c}{ Conventional orchards - locality } \\
\cline { 2 - 7 } & Dąbrowice & Maków & Mokra Lewa & Dąbrowice & Julków & Ostrowiec \\
\hline Total number of weed species & 38 & 44 & 63 & 24 & 18 & 28 \\
Number of short-lived species & 30 & 30 & 34 & 19 & 15 & 21 \\
Number of perennial species & 8 & 14 & 29 & 5 & 3 & 7 \\
Number of broad-leaved species & 30 & 38 & 53 & 20 & 15 & 24 \\
Number of grassy species (including sedges) & 7 & 5 & 9 & 3 & 3 & 3 \\
Mean number of species in phytosociological relevé & 19 & 21 & 24 & 11 & 12 & 16 \\
Mean total cover factor & 9278 & 9418 & 9867 & 9094 & 8262 & 9431 \\
Total cover factor of short-lived weeds & 7476 & 4740 & 4562 & 8101 & 7154 & 7538 \\
Total cover factor of perennial weeds & 1802 & 4678 & 5305 & 993 & 1108 & 1893 \\
Contribution of short-lived species in weed covering (\%) & 80.6 & 50.3 & 46.2 & 89.1 & 86.6 & 79.9 \\
Contribution of perennial species in weed covering (\%) & 19.4 & 49.7 & 53.8 & 10.9 & 13.4 & 20.1 \\
\hline
\end{tabular}


Table 8. Biodiversity of weed communities in organic and conventional apple orchards Dąbrowice, as an average for four years (2010-2013)

\begin{tabular}{lcc}
\hline \multicolumn{1}{c}{ Feature } & $\begin{array}{c}\text { Organic } \\
\text { orchard }\end{array}$ & $\begin{array}{c}\text { Conven- } \\
\text { tional } \\
\text { orchard }\end{array}$ \\
\hline $\begin{array}{l}\text { Mean number of weed species in } \\
\text { spring relevé }\end{array}$ & 21 & 13 \\
$\begin{array}{l}\text { Mean total number of weeds } \\
\text { (plants'm }{ }^{-2} \text { ) }\end{array}$ & 187.5 & 199.3 \\
$\begin{array}{l}\text { Shannon-Wiener's biodiversity in- } \\
\text { dex }- \text { H' }\end{array}$ & 2.343 & 1.686 \\
Simpson's dominance index - D & 0.141 & 0.259 \\
\hline
\end{tabular}

\section{DISCUSSION}

The synanthropic flora of orchards is formed under the conditions of a specific monoculture, which is characterized by long-term use, lack of tillage and crop rotation, frequent establishment of one orchard after another on specialized farms, and in the case of conventional orchards - regular use of herbicides as the only method of controlling weed infestation under tree crowns. For these reasons, the results of studies on orchards are difficult to compare with the results obtained for organic and conventional short-lived crops, for example, cereals and vegetables. Comparison of the species composition of the organic and conventional orchards has shown that weed species, such as Echinochloa crusgalli and Chenopodium album heavily infested both the organic orchards (all 3) and the conventional ones (2 of 3). Other short-lived species: Stellaria media, Poa annua and Capsella bursa-pastoris dominated in all the conventional orchards, which means that the use of postemergence herbicides contributes to their development at the expense of other species. Only one of these species, Stellaria media, played an important role in the organic orchard (Dąbrowice), which is undoubtedly connected with the succession of weed infestation. Indeed, the organic orchard in Dąbrowice was established in an area of long-term cultivation of fruit crops that had been treated with herbicides, and is an example of the conversion of conventional farming into organic crop growing. The role of herbicides in the compensation of weed infestation and 'promotion' of some species is proven by the fact that among the 25 weed species occurring in a young organic orchard in Skierniewice, with soil cultivation and mulching, there was no presence of Stellaria media, Capsella bursa-pastoris or Geranium pusillum, species typical for conventional orchards in the region, which were probably not competitive with vigorously growing weeds such as $\mathrm{Po}$ lygonum hydropiper, Amaranthus retroflexus, and Setaria pumila (Mika 2004). An important role in determining the species composition of weed communities is played by the time of tillage. Mechanical weeding of root crops during the weed-growing period reduces the occurrence of overwintering annual weeds and stimulates the growth of summer annual weeds (Elsen 2000). This relationship explains the presence in the assessed organic orchards of large numbers of species such as Echinochloa crus-galli, Chenopodium album, Amaranthus retroflexus, and Setaria pumila. Glyphosate - the basic herbicide used in conventional orchards was equally effective in reducing the number of broad-leaved weed species and grassy species (including sedges). This is not consistent with the results of assessments of the flora in arable fields, on the basis of which it was found that grasses are more resistant to the intensive methods of weed control than broad-leaved weeds (Moreby et al. 1994; Hald 1999; Rydberg \& Milberg 2000; Hyvönen et al. 2003).

In the organic orchards in Maków and Mokra Lewa, the proportion of short-lived and perennial weeds was similar, with Agropyron repens being an important species in both orchards. In all the conventional orchards and in the organic orchard in Dąbrowice, the soil was covered predominantly by short-lived weeds. The small share of Agropyron repens and other perennial weeds in the infestation of the organic orchard in Dąbrowice should be attributed to the succession of infestation and effective reduction in perennial weeds by repeated herbicide treatments in the past. The dominance of annual weeds in Dąbrowice confirms the thesis that the conversion of conventional farming into organic crop growing, after many years of herbicide use or frequent soil tillage, increases, to a limited extent, the biodiversity of weeds (Albrecht \& Mattheis 1998). The share of Agropyron repens in the infestation of the organic orchards increased with each passing year. The total absence of Agropyron repens 
in the conventional orchards confirms the thesis that the use of herbicides can completely eliminate some species from agrophytocoenoses (Rola \& Rola 2001). The proliferation of couch grass in the assessed organic orchards should be attributed to low effectiveness of eradication by a shallow-working tiller or other tools (Rabcewicz \& Białkowski 2011; Granatstein et al. 2014). Similar problems with the proliferation of Agropyron repens in organic orchards occur following the use of other ineffective methods of controlling it, such as organic (natural) herbicides/flaming, mulching, or mulching/flaming (Stefanelli et al. 2009; Granatstein et al. 2014).

The total number of weed species in each of the three organic orchards was higher than in the conventional orchards, which was likely a result of the way as the soil was maintained, more diverse environmental conditions, the surroundings of the orchard and succession. The assessed conventional orchards and the organic orchard in Dąbrowice were formerly a part of large complexes of orchards, whereas the orchards in Maków and Mokra Lewa were adjacent to field crops. The diversity of weeds in the organic orchards in Maków and Mokra Lewa may have been stimulated by crop rotation, which had been regularly conducted before the orchard was established (Derksen et al. 1993). One has to agree with the view that biodiversity is determined by a number of factors, not only the weed control method, environmental conditions, or crop rotation, but also the size and shape of the field, the type of neighboring crops and weed infestation in them, as well as the proximity of trees and natural habitats, which are, in their own way, random factors (Elsen 2000; Hole et al. 2005). Conclusive data on the effect of soil maintenance method on the diversity of weeds was provided by the comparison of the organic and conventional orchards in Dąbrowice. Biodiversity of weeds expressed by the number of species, Shannon-Wiener's biodiversity index (H') and Simpson's dominance index (D) was higher for the organic orchards than the conventional one. This confirms the results obtained in Bulgaria, where the number of weed species occurring in an organic orchard (16) was twice that found in a conventional orchard (8) (Tasseva 2005).

\section{CONCLUSIONS}

1. The biodiversity of weeds in the organic orchard was greater than in the conventional orchard.

2. Repeated shallow cultivation of the soil, without herbicide treatments, resulted in the proliferation of perennial weeds.

3. Species composition of weed infestation was modified by the method of soil cultivation, environmental conditions, and the succession of agrophytocoenoses.

\section{Acknowledgements}

The work has been supported by a grant under 'Development of innovative products and technologies for organic fruit production', co-financed from the EU Regional Development Fund through the Polish Innovation Economy Operational Programme (contract UDA-POIG. 01.03.0110-109/08-00).

\section{REFERENCES}

Albrecht H., Mattheis A. 1998. The effects of organic and integrated farming on rare arable weeds on the Forschungsverbund Agrarökosysteme München (FAM) research station in southern Bavaria. Biological Conservation 86: 347-356. DOI: 10.1016/s0006-3207(98)00028-7.

Anyszka Z., Kohut M. 2011. Biodiversity of segetal weed communities in cultivations of selected vegetable crops. Progress in Plant Protection/Postępy w Ochronie Roślin 51(3): 1219-1224. [in Polish with English abstract]

Błażewicz-Woźniak M. 2004. Primary weed infestation of carrot in conservation tillage. Acta Scientiarum Polonorum, Hortorum Cultus 3(1): 165-174. [in Polish with English abstract]

Braun-Blanquet J. 1964. Pflanzensoziologie. $3^{\text {rd }}$ ed. Springer-Verlag Vienna, 865 p. DOI: 10.1007/9783-7091-8110-2.

Derksen D.A., Lafond G.P., Thomas A.G., Loeppky H.A., Swanton C.J. 1993. Impact of agronomic practices on weed communities: tillage systems. Weed Science 49: 409-417.

Dobrzański A., Pałczyński J. 2007. The comparison of weed infestation of bean in organic and non-organic systems. Pamiętnik Puławski 145: 17-24. [in Polish with English abstract] 
van Elsen T. 2000. Species diversity as a task for organic agriculture in Europe. Agriculture, Ecosystems \& Environment 77: 101-109. DOI: 10.1016/s01678809(99)00096-1.

Granatstein D., Andrews P., Groff A. 2014. Productivity, economics, and fruit and soil quality of weed management systems in commercial organic orchards in Washington State, USA. Organic Agriculture 4: 197-207. DOI: 10.1007/s13165-014-0068-0.

Hald A.B. 1999. Weed vegetation (wild flora) of long established organic versus conventional cereal fields in Denmark. Annals of Applied Biology 134: 307314. DOI: 10.1111/j.1744-7348.1999.tb05269.x.

Hole D.G., Perkins A.J., Wilson J.D., Alexander I.H., Grice P.V., Evans A.D. 2005. Does organic farming benefit biodiversity? Biological Conservation 122: 113-130. DOI: 10.1016/j.biocon.2004.07.018.

Hyvönen T., Ketoja E., Salonen J., Jalli H., Tiainen J. 2003. Weed species diversity and community composition in organic and conventional cropping of spring cereals. Agriculture, Ecosystems \& Environment 97: 131-149. DOI: 10.1016/s01678809(03)00117-8.

Jędruszczak M., Antoszek R. 2004. Tillage systems and biodiversity of weed communities in winter wheat monoculture. Acta Scientiarum Polonorum, Agricultura 3(2): 47-59. [in Polish with English abstract]

Kuźniewski E., Jezierska-Domaradzka A. 2007. Weed infestation of root crops on chosen organic and conventional farms in Opole region. Progress in Plant Protection/Postępy w Ochronie Roślin 47(3): 182186. [in Polish with English abstract]

Lipecki J. 2004. Orchards weeds in Lublin region twenty years on - preliminary report. Journal of Fruit and Ornamental Plant Research 12: 105-111, Special ed.

Lisek J. 2012. Synanthropic orchard flora in West Mazovia - central Poland. Journal of Fruit and Ornamental Plant Research 20(2): 71-83. DOI: 10.2478/v10290-012-0017-5.

Marshall E.J.P., Brown V.K., Boatman N.D., Lutman P.J.W., Squire G.R., Ward L.K. 2003. The role of weeds in supporting biological diversity within the crop fields. Weed Research 43: 77-89. DOI: 10.1046/j.1365-3180.2003.00326.x.

Mashaly I.A., Awad E.R. 2003. Weed flora of orchards in the Nile delta, Egypt: floristic features. Asian Journal of Plant Sciences 2(3): 314-324. DOI: 10.3923/ajps.2003.314.324.

Mika A. 2004. The importance of biodiversity in natural environment and in fruit plantations. Journal of
Fruit and Ornamental Plant Research 12: 11-21, Special ed.

Moreby S.J., Aebischer N.J., Southway S.E., Sotherton N.W. 1994. A comparison of the flora and arthropod fauna of organically and conventionally grown winter wheat in southern England. Annals of Applied Biology 125: 13-27. DOI: 10.1111/j.17447348.1994.tb04942.x.

Rabcewicz J., Białkowski P. 2011. The efficiency of mechanical weed control in ecological apple production. Journal of Research and Applications in Agricultural Engineering 56(4): 79-83. [in Polish with English abstract]

Rola H., Rola J. 2001. Positive and negative aspects of herbicide application in agricultural crops in Poland (1950-2000). Progress in Plant Protection/Postępy w Ochronie Roślin 41(1): 47-57. [in Polish with English abstract]

Rydberg N.T., Milberg P. 2000. A survey of weeds in organic farming in Sweden. Biological Agriculture \& Horticulture 18: 175-185. DOI: 10.1080/01448765.2000.9754878.

Sekutowski T., Domaradzki K. 2009. Biodiversity of weed species in winter wheat monoculture caused by reduced of tillage. Fragmenta Agronomica 26(4): 160-169. [in Polish with English abstract]

Shannon C.E. 1948. A mathematical theory of communication. Bell System Technical Journal 27(3): 379423. DOI: 10.1002/j.1538-7305.1948.tb01338.x; 27(4): $\quad 623-656 . \quad$ DOI: $\quad 10.1002 / \mathrm{j} .1538-$ 7305.1948.tb00917.x.

Simpson E.H. 1949. Measurement of diversity. Nature 163: 688 .

Stefanelli D., Zoppolo R.J., Perry R.L., Weibel F. 2009. Organic orchard floor management systems for apple effect on rootstock performance in the Midwestern United States. HortScience 44(2): 263267.

Tasseva V. 2005. Species composition of weed vegetation in different apple growing technologies. Notulae Botanicae Horti Agrobotanici Cluj-Napoca 25: 59-64.

Ustuner T., Ustuner M. 2011. Investigation on different mulch materials and chemical control for controlling weds in apple orchard in Turkey. Scientific Research and Essays 6(19): 3979-3985. DOI: 10.5897/SRE10.867.

Wróbel M. 1999. Orchard flora in Szczecin surroundings. Journal of Fruit and Ornamental Plant Research 7(3): 133-145. 